\title{
Violence viewed by psychopathic murderers
}

\author{
Adapting a revealing test may expose those psychopaths who are most likely to kill.
}

Ds sychopathic murderers are often portrayed as cold-blooded, emotionless and lacking in remorse ${ }^{1}$, but they are also adept at lying and at feigning the emotions in which they are deficient. Here we adapt a test known as the Implicit Association Test (IAT) ${ }^{2}$, which was previously used to assess concealed prejudices, to show that psychopathic murderers have abnormal cognitive associations regarding violence, which may underpin their actions. Such implicit measures may provide us with an important insight into the criminal mind.

The ability of psychopaths to con, lie and manipulate means that their potential danger to others may not be appreciated by criminal-justice agencies, that their response to clinical assessment is often rigged $^{3}$, and that researchers are blinded to underlying motives (Fig. 1). Faced with the problem of detecting socially stigmatic beliefs, psychologists have developed tasks that measure these attitudes implicitly. The IAT has successfully quantified beliefs that people may wish to disguise, such as negative views about racial groups, homosexuality or obesity. We have adapted the IAT to reveal implicit beliefs about violence in psychopathic murderers.

We followed the general methodology of the IAT ${ }^{2}$. Briefly, uppercase words (for example, 'UGLY') are classified as being 'pleasant' or 'unpleasant', and lowercase words (for example, 'kill') are classified as 'violent' or 'peaceful', by pressing corresponding buttons. When the same response key is assigned for both the unpleasant and

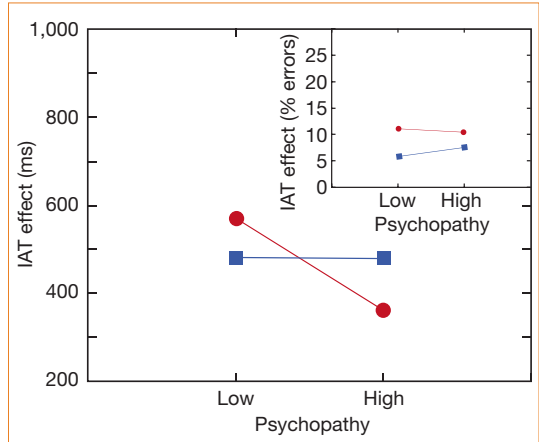

Figure 2 Results of the Implicit Association Test (IAT) effect (difference in reaction time between incongruent and congruent conditions; see text), plotted as a function of psychopathy score $\left(\mathrm{PCL}-\mathrm{R}^{4}\right)$ for murderers (red) and non-murderers (blue). Inset, IAT effect for error rates. All participants gave written informed consent. We used eight 'pleasant' and eight 'unpleasant' words ${ }^{9}$, and eight 'violent' and eight 'peaceful' words. Both the congruent and incongruent blocks consisted of 64 trials, with words presented in a pseudo-random order. Further details are available from the authors. violent words (this is termed the congruent condition), most people find the task easy. But when pleasant and violent words share the same response key (the incongruent condition), most people find this confusing. The association between 'pleasant-unpleasant' and 'violent-peaceful' is indexed by means of the IAT effect (reaction time for the incongruent condition minus reaction time for the congruent condition).

Our sample $(n=121)$ was recruited from consecutive admissions to a secure therapeutic community for male offenders with personality disorders. None of the participants had a co-morbid mental illness and none was taking any illicit drug or medication. Psychopathy was measured using the Psychopathy Checklist-Revised test ${ }^{4}$; those who scored over 25 were labelled as psychopaths ${ }^{5}$. We selected the murderers in order to target the most seriously violent group. Our final groups consisted of 13 psychopathic murderers, 17 non-psychopathic murderers, 39 psychopathic other offenders and 52 non-psychopathic other offenders. There was no significant difference in intelligence quotient (IQ) among the groups.

The results from the 'violent' IAT test are shown in Fig. 2. Statistical analysis (analysis of variance) indicates that there was no significant effect due to grouping (murderer versus other offender) or psychopathy score. Crucially, the interaction between these variables was significant $(P<0.05)$ and remained so even when age and IQ (as assessed by the revised National Adult Reading Test $^{6}$ ) were added as covariates. As expected, the psychopathic murderers showed a much lower IAT effect than the non-psychopathic murderers $(P<0.05)$. There was no significant difference in error rate related to offence type, psychopathy or interaction between these variables (Fig. 2, inset). A control IAT (flower-insect; pleasant-unpleasant) revealed no effect for psychopathy or offence type.

Our results indicate that the reduced violent-IAT effect seen in psychopathic murderers is likely to be due to their abnormal beliefs about violence, rather than to some other nonspecific effect such as poor impulse control and/or deficits in decisionmaking. Psychopathic murderers have diminished negative reactions to violence compared with non-psychopathic murderers and other offenders.

To our knowledge, this is the first cognitive demonstration of abnormal social beliefs in murderous psychopaths. The interaction between psychopathy and murder suggests that it is the combination of these two factors

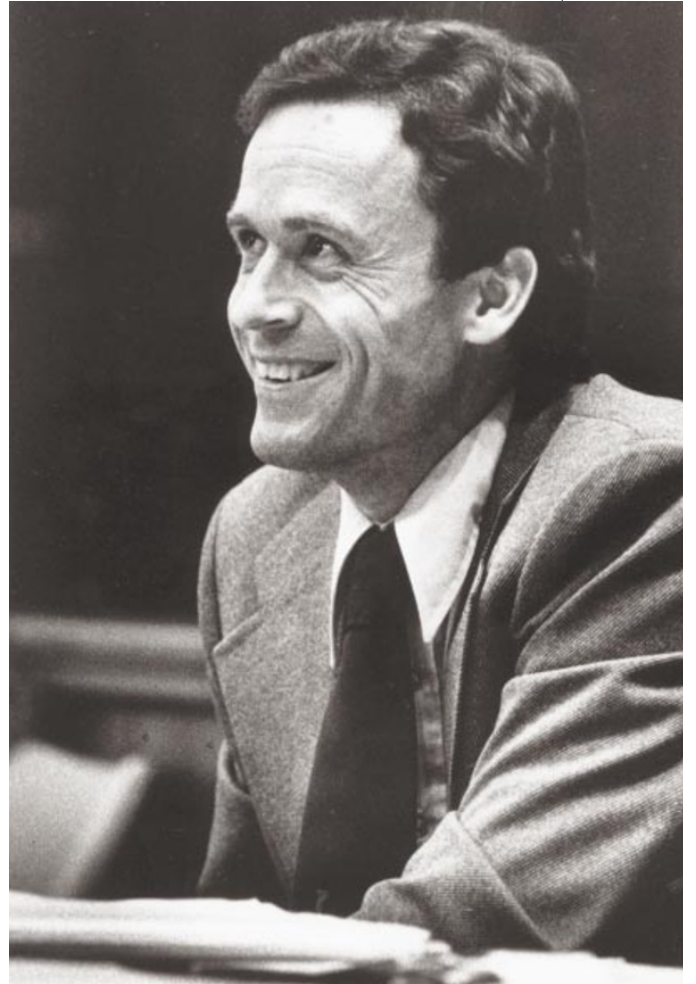

Figure 1 Portrait of a serial killer: psychopathic murderers such as Ted Bundy can often project a deceptively charming persona.

that is crucial in identifying deficient implicit beliefs about violence, resulting in a relatively rare subpopulation of very violent psychopaths. Obviously, not all murderers have similar motivations or beliefs about violence, and not all psychopaths commit murder (as illustrated by the phenomenon of the 'whitecollar' psychopath ${ }^{7}$ ).

Our results have some testable implications. For example, non-psychopaths could become sensitized to violence after committing murder $^{8}$ (hence their greater scores in the IAT). The difference between the psychopathic groups may reflect two separate, stable populations of psychopathic offenders: one with deficient social beliefs (and therefore an increased disposition towards extreme violence) and the other in which such negative beliefs are absent. If this difference can be picked up by the 'violent' IAT before an offence is committed, this test may become an important tool for distinguishing psychopaths who are likely to commit extremely violent offences from those who are not.

Nicola S. Gray ${ }^{\star} \dagger$,

Malcolm J. MacCulloch ${ }^{\star} \ddagger$, Jennifer Smith ${ }^{\star}$, Mark Morris $₫$, Robert J. Snowden*

*School of Psychology, Cardiff University,

Cardiff CF10 3YG, UK 
e-mail: grayns@cardiff.ac.uk

$\dagger$ South Wales Forensic Psychiatric Service,

Caswell Clinic, Glanrhyd Hospital,

Mid Glamorgan CF36 4LN, UK

$\ddagger$ Department of Psychological Medicine,

University of Wales College of Medicine, Heath

Park, Cardiff CF14 4XN, UK

$\$ H M$ Prisons, Grendon and Springhill,

Grendon Underwood, Aylesbury,

Buckinghamshire HP18 OTL, UK

1. Cleckley, H. The Mask of Sanity (Mosby, St Louis, 1941).

\section{Prion diseases}

\section{BSE in sheep bred for resistance to infection}

elective breeding for disease-resistant genotypes is being pursued as a means of eradicating scrapie (and bovine spongiform encephalopathy (BSE), if it is present) from sheep flocks. Here we show that the genotype associated with the highest resistance can still be infected with BSE by intracerebral inoculation. Although the relevance of this finding to sheep exposed to natural infection remains to be determined, it may have important implications for disease-eradication strategies.

Susceptibility and resistance to transmissible spongiform encephalopathies (TSEs) in sheep is largely controlled by polymorphisms at codons 136, 154 and 171 of the gene that encodes prion protein $(\operatorname{PrP})$. Representing the resulting alleles as the amino acids at these positions ${ }^{1}$, susceptibility to scrapie is strongly associated with valine $(\mathrm{V})$ at position 136 and/or glutamine (Q) at position 171, and susceptibility to experimental BSE is associated predominantly with the alanine-arginine-glutamine (ARQ) allele ${ }^{2}$.

The $A R R$ allele is associated with resistance to natural scrapie - the disease is rare in $A R R$ heterozygotes ${ }^{3}$ and only one unconfirmed case has ever been reported in a homozygote ${ }^{4}$. The limited data available suggest that $A R R / A R R$ sheep are also resistant to experimental oral challenge with scrapie and $\mathrm{BSE}^{5,6}$. Britain has therefore initiated the National Scrapie Plan, which aims eventually to eradicate scrapie (as well as any BSE concealed in sheep $)^{7}$ by means of increasing the frequency of the $A R R$ allele and decreasing that of the $V R Q$ allele.

We investigated whether $A R R / A R R$ sheep are still resistant to transmission of BSE when inoculated intracerebrally with infected cattle-brain homogenate $(0.05 \mathrm{~g})$. Groups of New Zealand (scrapie-free) sheep of three different breeds (Suffolk, Cheviot and Poll Dorset) and six different $\operatorname{PrP}$ genotypes (VRQ/VRQ, VRQ/ARQ, VRQ/ARR, ARQ/ARQ, ARQ/ARR and $A R R / A R R)$ were tested.
2. Greenwald, A. G., McGhee, J. L. \& Schwartz, J. L. J. Person. Soc. Psychol. 74, 1464-1480 (1998).

3. Rice, M. E., Harris, G. T. \& Cormier, C. A. Law Hum. Behav. 16, 399-412 (1992)

4. Hare, R. D. The Hare Psychopathy Checklist Revised (MultiHealth Systems, Toronto, 1991).

5. Cooke, D. L. \& Michie, C. J. Abnorm. Psychol. 108, 58-68 (1999).

6. Nelson, H. National Adult Reading Test (NART) Manual (NFER-Nelson, Windsor, 1982).

7. Hare, R. D. Without Conscience (Guilford, New York, 1999).

8. Gray, N. S. et al. J. Forens. Psychiat. Psychol. 14, 27-43 (2003).

9. Bellezza, F. S., Greenwald, A. G. \& Banaji, M. R. Behav. Res. Methods Instr. Comp. 18, 299-303 (1986).

Competing financial interests: declared none.

As expected, the incubation period (495-671 days) was shortest in ARQ/ARQ sheep, with 17 out of 19 being affected. So far, four out of ten VRQ/VRQ and two out of ten VRQ/ARQ Cheviot sheep have also succumbed to disease after incubation periods of 881-1,092 days. Unexpectedly, 3 out of 19 BSE-challenged $A R R / A R R$ sheep also showed clinical symptoms after incubation periods of $1,008,1,124$ and 1,127 days, respectively, but none of the $A R R$ heterozygotes has been clinically affected to date.

Western-blot analysis of the abnormal protease-resistant fraction of $\operatorname{PrP}\left(\mathrm{PrP}^{\mathrm{Sc}}\right)$ (results not shown) and immunohistochemical labelling of disease-associated PrP in brain sections from the $A R R / A R R$ sheep (Fig. 1) were consistent with experimental $\mathrm{BSE}$ in $A R Q / A R Q$ sheep $^{8,9}$. This contrasts with the results of in vitro cell-free conversion experiments, in which conversion of the sheep ARR PrP protein to $\operatorname{PrP}^{\mathrm{Sc}}$ was extremely inefficient ${ }^{10}$.

The susceptibility of $A R R / A R R$ sheep to intracerebral injection with BSE indicates that these animals cannot be regarded as having absolute genetic resistance to TSE infection. The significance of this result for sheep that are exposed to natural TSE infection remains to be determined. In ongoing experiments, $A R R / A R R$ sheep orally dosed with BSE have remained free from clinical disease or evidence of preclinical infection for up to 2,100 days after BSE challenge (ref. 6

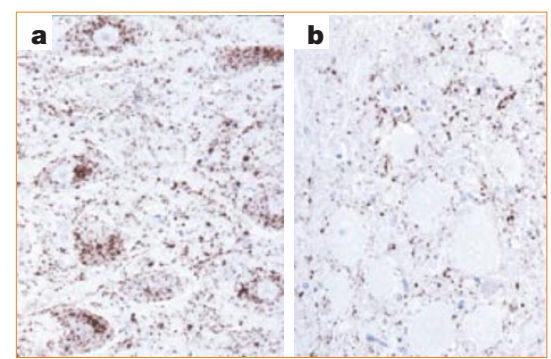

Figure 1 Immunohistochemical labelling of abnormal PrP protein in the dorsal motor nucleus of the vagus of a sheep that is homozygous for the ARR allele (see text) and has been infected with bovine spongiform encephalopathy (BSE). a, Intraneuronal and neuropil-associated accumulations are revealed by the R145 antibody; b, the antibody P4 reveals neuropil-associated (but not intraneuronal) extracellular accumulation. This pattern of labelling can distinguish BSE from scrapie in sheep. and J.F., unpublished results). However, the number of animals in these studies is probably too small to rule out susceptibility even if the surviving animals remain free of infection.

Our results may reflect those found for pigs, in which no natural case of BSE has been reported. Pigs inoculated with BSE simultaneously by intracerebral and peripheral routes developed TSE within 520-1,130 days, whereas those exposed to repeated oral challenge remained healthy for up to 2,550 days after infection ${ }^{11}$.

Even if $A R R / A R R$ sheep exposed to natural TSE infection do not succumb to disease, they might be able to act as subclinical carriers of infection. However, no abnormal PrP (and, by implication, infectivity) was detected in the lymphoid tissues of any of the ARR/ARR sheep cases of BSE reported here. Likewise, neither clinically normal $A R R / A R R$ sheep in scrapie-endemic flocks, nor those challenged orally with TSEs, show evidence of abnormal PrP deposition in peripheral tissues although here again the number of animals examined is small ${ }^{6,12}$. These results indicate that $A R R / A R R$ sheep are unlikely to excrete the infectious agent, although transmission of infection to in-contact animals in the absence of detectable abnormal PrP cannot be ruled out.

We have shown that $A R R / A R R$ sheep can become infected when inoculated intracerebrally with a high dose of BSE, but there is, as yet, no evidence to suggest that they are susceptible when exposed by natural routes of infection. But the fact that the ARR PrP protein can undergo conversion to a pathological isoform in vivo raises questions about the basic mechanisms that underpin genetic resistance to TSEs.

Fiona Houston ${ }^{\star}$, Wilfred Goldmann $\dagger$, Angela Chong $\dagger$, Martin Jeffrey市, Lorenzo Gonzálezł, James Foster $\dagger$, David Parnham $\dagger$, Nora Hunter $\dagger$

${ }^{\star}$ Institute for Animal Health, Compton, Newbury, Berkshire RG20 7NN, UK

e-mail: fiona.houston@bbsrc.sc.uk

$\dagger I A H$ Neuropathogenesis Unit, Ogston Building, Edinburgh EH9 3JF, UK

\$Veterinary Laboratories Agency Lasswade, Pentlands Science Park, Penicuik, Midlothian EH26 OPZ, UK

\footnotetext{
1. Hunter, N. Trends Microbiol. 5, 331-334 (1997).

2. Goldmann, W., Hunter, N., Smith, G., Foster, J. \& Hope, J. J. Gen. Virol. 75, 989-995 (1994).

3. van Keulen, L. J. et al. J. Clin. Microbiol. 34, 1228-1231 (1996)

4. Ikeda, T. et al. J. Gen. Virol. 76, 2577-2581 (1995)

5. O’Rourke, K. I. et al. J. Gen. Virol. 78, 975-978 (1997).

6. Jeffrey, M. et al. J. Comp. Pathol. 124, 280-289 (2001).

7. Kao, R. R. et al. Science 295, 332-335 (2002).

8. Hope, J. et al. J. Gen. Virol. 81, $851(2000)$

9. Jeffrey, M. et al. J. Comp. Pathol. 125, 271-284 (2001).

10. Bossers, A., de Vries, R. \& Smits, M. A. J. Virol. 74, 1407-1414 (2000).

11. Ryder, S. J., Hawkins, S. A., Dawson, M. \& Wells, G. A. J. Comp. Pathol. 122, 131-143 (2000).

12. Jeffrey, M. et al. J. Comp. Pathol. 127, 264-273 (2002).

Competing financial interests: declared none.
} 